\title{
Opportunistic Scheduling of a Message Ferry in Sensor Networks
}

\author{
Veeraruna Kavitha \\ INRIA, Sophia Antipolis, France \\ LIA, University of Avignon, France \\ Kavitha.Voleti_Veeraruna@sophia.inria.fr
}

\author{
Eitan Altman \\ INRIA, Sophia Antipolis, France \\ Eitan.Altman@sophia.inria.fr
}

\begin{abstract}
We study the concept of Ferry based Wireless Local Area Network (FWLAN), in which a number of isolated nodes are scattered over some area and where communication between a node and the outer world, or communication between the nodes, are made possible via a message ferry. The Ferry travels in a predetermined cyclic path and collects/delivers messages from/to a node when it stops in the vicinity of the node. The radio conditions may change between successive visits of a station. We consider an opportunistic service in which the shuttle serves a node only if its radio conditions are good. Our analytical results are based on the theory of polling systems with rerouting.
\end{abstract}

\section{Keywords}

Polling systems, Wireless LAN

\section{Categories and Subject Descriptors}

C.2 [Computer-communication networks]: Local and Wide-Area Networks, Network Architecture and Design

\section{General Terms}

Performance

\section{INTRODUCTION}

Message Ferries are mobile relays that serve as "postman" to deliver/collect messages to/from static or dynamic wireless nodes. Mobile BS have been proposed in the context of mobile Ad Hoc Networks [10], of Vehicular Ad-Hoc Networks (Vanets) [6] and of wireless sensor networks [7]. In the UmassDiesel project, computers have been installed in 30 out of 40 buses which serve as Message Ferry to deliver messages to throw boxes (http://prisms.cs.umass.edu/diesel).

\footnotetext{
${ }^{*}$ This project was supported by the Indo-French Centre for the Promotion of Advanced Research (IFCPAR), project 4000-IT-1 and by the INRIA association program DAWN. The french co-author is supported by the Bionets European project.

Permission to make digital or hard copies of all or part of this work for personal or classroom use is granted without fee provided that copies are not made or distributed for profit or commercial advantage and that copies bear this notice and the full citation on the first page. To copy otherwise, to republish, to post on servers or to redistribute to lists, requires prior specific permission and/or a fee.

MobiOpp '10, February 22-23, 2010, Pisa, Italy.

Copyright 2010 ACM 978-1-60558-925-1/10/02 ...\$10.00.
}

We are concerned with a message ferry that serves as a mobile access point in a local area network which we call FWLAN (Ferry Wireless LAN); the ferry delivers and collects messages from the nodes on some geographic area $\Delta$. The nodes are mobile but move rather slowly in comparison with the ferry. There is a fixed base station (BS) that is connected to the global Internet (or to other BS) and thus enables communication between nodes in the FWLAN and the outer world. The ferry brings all traffic from (respectively to) nodes in the FWLAN to (resp. from) the BS.

The ferry moves in a cyclic route periodically and stops at finite number of predetermined stops. Each stop is assigned an area that contains all points closer to that stop than to other stops. Upon reaching a stop, the ferry attempts serving all the active nodes of the assigned area, one after the other and independent of the rest of the other active nodes, with a probability that depends upon the node's (time varying radio) fading factor. We call this probability, which is a design parameter, the Oppurtunistic scheduling policy of the ferry. At every stop, it first serves the downlink nodes (i.e., dumps the data collected from the BS to the nodes) and then attends the uplink nodes (i.e., collects the data from the nodes to be delivered to the BS).

This kind of system can best be studied using a polling system, wherein a ferry serves a finite number of queues in a cyclical order $[5,3,9,8]$. The main contribution of this paper is to map this problem into a polling system. We obtain the capacity of the system and identify a policy that achieves it. We derive an explicit expression for the expected virtual workload in the system as a function of the scheduling policy of the server. We then formulate the optimal choice of opportunistic scheduling policy as mathematical program.

\section{SYSTEM MODEL AND NOTATIONS}

We consider a geographical area $\Delta$ in which nodes (sensors) are scattered. The nodes are mobile, move to an independent location in one cycle time of the ferry but their movement is slow enough that they remain in the vicinity of the previous location. We assume that the network is sparse and there is no direct global connectivity. In order to receive messages from the nodes or to send messages to them, a ferry called "message ferry" or "message shuttle" moves around and serves as a postman. The nodes either generate data to or acquire data form nodes within and/or outside the area. In order to route the data to and from outside the area, the shuttle has to pass through a BS that serves as a gateway.

Ferry's Route : The ferry moves in a closed path repeat- 
edly and stops at the same finite number, $K$, of predetermined stops in every cycle. The area is divided into $K$ disjoint subareas and each stop is associated with one of the subareas. A node belongs to that subarea if and only if its signal is strongest at the associated Ferry stop in comparison with the other stops. At each stop, the ferry serves all the nodes located in the associated subarea.

Let $\left\{Q_{1}, Q_{2}, \cdots, Q_{K}\right\}$ represent the location of stops of the ferry. For each $i$ let $I_{i}$ represent the subarea associated with stop $i$. Indexing is done in a circular manner.

We assume that $B S$ is located at $Q_{1}$ and that $I_{1}$ contains only the $\mathrm{BS}^{1}$. The radio connection between the ferry and $\mathrm{BS}$ are assumed to be very good and hence one can neglect the time taken by the ferry for serving the BS.

While traversing through the path, at every stop $i$, the ferry observes the general channel conditions of each and every active node. It then decides either to serve a node indepedently of the others based on its fading conditions. It first downloads all the data collected from the BS destined to the nodes, oppurtunistically, located in area $I_{i}$ and then collects all the uplink data messages (once again based on their fading factors) that have arrived since its last visit. It continues collecting the messages till there are no more uplink data messages in $I_{i}$ (with good fading conditions).

Arrival process: We consider traffic generated at the nodes which we call "uplink", and traffic that arrives to the nodes which is called "downlink". We define the exogenous uplink (downlink) Poisson point processes $\left\{T_{n}, L_{n}\right\}$ with rate $\lambda^{u}$ $\left(\lambda^{d}\right)$, where $L_{n}$, the mark associated with Poisson process represents the source or destination node. Every transmission consists of transfer of fixed $\eta$ number of bytes.

Radio channel and the service time: Ferry uses a wireless link to serve the customers. We assume that the radio channel between a mobile and the Ferry can be described as the product of some random part (that changes in time, space in a random way) and some deterministic part (that does not change in time). The deterministic part corresponds to the path loss and is determined by the distance $d$. The random part may corresponds to slow fading (e.g. shadowing). The ferry can transfer the messages from/to the nodes at a distance $d$ from it, at a rate

$$
R_{s f}(d, \Phi)=r(d) \times \Phi,
$$

where $\Phi$ is a discrete valued random radio gain factor at the instant that the ferry approaches the station and $r(d)$ is some decreasing function that represents the gain at distance $d$. The random factor $\Phi$ depends upon the location of the node and the time of transmission. It is an indepedent process across nodes as well as from one cycle to another. It is however assumed to remain constant during the entire transmission period. The service time, i.e., the time required to transfer $\eta$ bytes, when the source/destination is located at $l \in I_{i}$ will be equal to,

$$
B(l, \phi)=\frac{\eta}{r\left(\left\|Q_{j}-l\right\|\right) \phi}
$$

Throughout the paper ||.|| represents either the area (length) of the two (one) dimensional region or the distance between two points. Let $\Phi \in\left\{\phi_{1}, \phi_{2}, \cdots,\right\}$ where $\phi_{1}>\phi_{2} \cdots$ and let $\pi_{i}:=\operatorname{Prob}\left(\Phi=\phi_{i}\right)$.

\footnotetext{
${ }^{1}$ That is the physical distance between $Q_{1}$ and $Q_{2}$ is zero. It is viewed as a seperate stop to simplify the analysis.
}

Opportunistic Scheduling: An opportunistic scheduler $v^{j}$ maps each possible state $\phi$ to a real number in the interval $[0,1]$. When serving a node in station $j$ the ferry measures the state $\phi$ of the node and then serves its request with probability $v^{j}(\phi)$. If not served, the packets have to wait till the next cycle to be considered again for transmission. Currently we consider a scheduler with $v^{j}=v$ for all $j$ but one can easily extend the results to general case.

At every station $j$, the ferry first attempts downloading the downlink data that it collected when it last visited the base station (and also the downlink data that is not transfered during the previous cycle), to each one of the destined nodes, which undergo fading $\phi^{d}(L)$, with probabilities $v\left(\phi^{d}(L)\right)$. After downlink, it collects the uplink data from each of the active nodes in $I_{j}$ in an exahustive way, independent of each other with probabilities, which once again depend upon the node's fading factor and the scheduler.

Walking times: After serving all the nodes in a stop $Q_{i}$, the ferry walks to the next stop $Q_{i+1}$. The walking time is $c_{1}\left\|Q_{i}-Q_{i+1}\right\|+c_{2}$, for some appropriate constants $c_{1}$, $c_{2}$. The constant $c_{2}$ represents the extra time for acceleration/deceleration while $c_{1}$ represents the speed of the ferry.

\section{REDUCING TO POLLING MODEL}

We model the FWLAN as a polling system with $\sigma:=$ $4 K+1$ queues and analyze its performance using the theory of Siddi etal's poling systems with rerouting ([8]). Any polling system consists of a number of queues served by a single server in a cyclic/periodic manner. Various types of polling systems are studied in literature: each differing from the other either in terms of the order in which the server serves the queues or in the service policies at each queue or in the number (finite, countably infinite or continuum) of queues etc. Below we show how FWLAN can be mapped to a particular polling system and also discuss the exact configuration of the polling system that models it.

Stop $Q_{j}$ modeled as four queues : As a first step, we model each stop, $Q_{i}$, as 4 independent queues, the first two numbered $4 j-3,4 j-2$ are for downlink while the next two numbered $4 j-1,4 j$ are for uplink. The queues $4 j-2,4 j$ do not offer any service (hence have zero serive times) and are only meant to represent delay of service to the next cycle, while the queues $4 j-3$ and $4 j-1$ carry out the actual services. That is, the users whose request will be served in the current cycle are viewed as if waiting in the queues $4 j-3$ for downlink service and $4 j-1$ for uplink service. The remaing users wait in queue $4 j-2$ (or $4 j$ if for uplink) to be rerouted by the ferry upon its arrival in station $j$ to either the same queue or to the queues $4 j-3(4 j-1)$ based on their fading factor in the next cycle and the oppurtunistic scheduler $v$. Further at the BS, i.e., at stop $Q_{1}$ (which handles only BS) has one queue, numbered $\sigma=4 K+1$, meant to assist downlink service.

Modeling of Oppurtunistic Uplink: The arrival of an uplink request (given by Poisson process at rate $\lambda^{u}$ ) brings along with it the mark $L$, the location of the source. If $L \in I_{j}$, then the arrival is either modeled to arrive at queue $4 j-1$ if the oppurtunistic scheduler $v$ decides to serve it the immediate next time the ferry arrives at stop $j$ (this decision is based on the fading factor at location $L$ and at the time the ferry arrives at stop $j$ ) or in the other condition at 
queue $4 j$. If the ferry does not serve the uplink request in the current cycle, the request has to wait for the next cycle. When the ferry arrives again at stop $j$ in the next cycle, it will oppurtunistically serve the user based on its the then fading factor. This process is modeled by the rerouting of the user from queue $4 j$ (the customers of which are assumed to come with zero service times) by the server of the equivalent polling system either to itself or to the other queue $4 j-1$ and by serving the queue $4 j$ using gated service. The queue $4 j-1$ is served using exhaustive discipline and there is no rerouting from this queue, i.e., the users leave the system after service in this queue. The first two moments of the service times of the customers in this queue is

$$
\begin{aligned}
b_{4 j-1} & :=\sum_{i} \frac{\eta \pi_{i}}{\phi_{i}} \frac{v\left(\phi_{i}\right)}{\sum_{l} \pi_{l} v\left(\phi_{l}\right)} E\left[\frac{1}{r\left(|| L-Q_{j} \|\right)} \mid L \in I_{j}\right] \\
b_{4 j-1}^{(2)} & :=\sum_{i} \frac{\eta^{2} \pi_{i}}{\phi_{i}^{2}} \frac{v\left(\phi_{i}\right)}{\sum_{l} \pi_{l} v\left(\phi_{l}\right)} E\left[\frac{1}{r\left(\left\|L-Q_{j}\right\|\right)^{2}} \mid L \in I_{j}\right]
\end{aligned}
$$

By the time the ferry arrives at stop $j$ in the next cycle, the user with unserved request is assumed to have moved to an independent location but in $I_{j}$ itself, i.e., the users in one cycle time move to a location independent of their previous location $L$ but their movement is slow enough that they still remain in $I_{j}$. This assumption ensures that the overall service times $B(L, \Phi)$ are independent across cycles, queues etc. The upload to BS of all the data collected by Ferry in every cycle is assumed to be immediate and is not considered for analysis.

For each $j$, the uplink queues $4 j-1,4 j$ have Poisson arrivals respectively with rates $\lambda_{4 j-1}:=\lambda^{u} l_{j} p_{s}, \lambda_{4 j}:=$ $\lambda^{u} l_{j}\left(1-p_{s}\right)$ where $l_{j}:=\operatorname{Prob}\left(L \in I_{j}\right)$ is the probability that a request arrives in area $I_{j}$ and $p_{s}:=\sum_{i} \pi_{i} v\left(\phi_{i}\right)$ is the probability that the request is scheduled in the coming cycle. The queues $4 j-1,4 j$ also have rerouting arrivals but only from queue $4 j$ according to the probabilities: $p_{4 j, 4 j-1}=p_{s}$, $p_{4 j, 4 j}=1-p_{s}$ and $p_{4 j-1,4 j}=p_{4 j-1,4 j-1}=0$. The probability of leaving the system after a queue, $p_{4 j-1,0}=1=$ $1-p_{4 j, 0}$.

Modeling of Oppurtunistic Downlink: Download from BS to ferry of all the downlink data is immediate. The ferry attempts delivering in current cycle only those packets that it collected just before it left the BS previously. This service is similar to globally gated service of polling systems and we model this situation via the queue $\sigma$ at BS whose service times are zero and from which the customers are rerouted to the appropriate downlink queues when the ferry arrives at $Q_{1}$ : the request is rerouted to queue $4 j-3$ if oppurtunistic scheduler would have assigned the ferry to download data immediately, i.e. in the cycle that is going to start immediately or to queue $4 j-2$ if the request has to wait for the next cycle.

The average service times of the customers in queue $4 j-3$ is same as that of $4 j-1$, i.e., $b_{4 j-3}=b_{4 j-1}$ and so is $b_{4 j-3}^{(2)}=$ $b_{4 j-1}^{(2)}$. All downlink Poisson arrivals occur in queue $\sigma$ (located at BS) with rate $\lambda^{d}$ and this queue has no rerouting arrivals. The remaining downlink queues have only rerouting arrivals according to the rerouting probabilities:

$$
\begin{array}{lll}
p_{\sigma, 4 j-3}=p_{s} l_{j}, & p_{\sigma, 4 j-2}=\left(1-p_{s}\right) l_{j}, & p_{\sigma, 0}=0 \\
p_{4 j-2,4 j-3}=p_{s}, & p_{4 j-2,4 j-2}=\left(1-p_{s}\right), & p_{4 j-2,0}=0 \\
p_{4 j-3,4 j-3}=0, & p_{4 j-3,4 j-2}=0, & p_{4 j-3,0}=1 .
\end{array}
$$

The $\sigma=4 K+1$ queues are served cyclically by the ferry in the order $1 \mapsto 2 \cdots \sigma \mapsto 1 \cdots$. Only the queues $\{4 j-$ $1\}$ experience the exhaustive service, while the rest of the queues experience gated service. The rerouted customers will be served when the ferry/server arrives at the queue to which the customer is rerouted the first time after rerouting. Walking times: in the equivalent polling system are zero between the pair of queues belonging to the same stop and equal that of walking distance between the correspond stops for the remaing pair of queues, i.e., $w_{4 j-3}, w_{4 j-2}, w_{4 j-1}=0$ and $w_{4 j}=c_{1} \| Q_{j}-Q_{j+1}+c_{2}$. Further, $w_{\sigma}=0$ (as the two stops $Q_{1}, Q_{2}$ are physically at the same place).

So, the Oppurtunistic FWLAN is mapped to a $\sigma$ polling system of [8] with mixed service desciplines. We obtain its performance like stability, virtual workload using [8].

\subsection{Stability and Capacity}

The total arrival rates at a queue $\left\{\gamma_{i}\right\}$ satisfy for all $j$ (see equation 2.1 of [8] for details) :

$$
\begin{aligned}
\gamma_{4 j-1} & =\lambda_{4 j-1}+\sum_{i=1}^{\sigma} \gamma_{i} p_{i, 4 j-1}=\lambda_{4 j-1}+\gamma_{4 j} p_{4 j, 4 j-1}, \\
\gamma_{4 j} & =\lambda_{4 j}+\sum_{i=1}^{\sigma} \gamma_{i} p_{i, 4 j}=\lambda_{4 j}+\gamma_{4 j} p_{4 j, 4 j}
\end{aligned}
$$

and hence by solving the above equations (for all $j$ ):

$$
\begin{aligned}
\gamma_{\sigma} & =\lambda^{d}, \quad \gamma_{4 j-3}=\lambda^{d} l_{j}, \quad \gamma_{4 j-1}=\lambda^{u} l_{j} \\
\gamma_{4 j-2} & =\frac{\lambda^{d} l_{j}\left(1-p_{s}\right)}{p_{s}} \text { and } \gamma_{4 j}=\frac{\lambda^{u} l_{j}\left(1-p_{s}\right)}{p_{s}} .
\end{aligned}
$$

Total network utilization factor $\rho:=\sum_{i} \gamma_{i} b_{i}$ equals ([8]),

$$
\left(\lambda^{u}+\lambda^{d}\right) \eta E\left[\sum_{j=1}^{K} \frac{1_{\left\{L \in I_{j}\right\}}}{r\left(|| L-Q_{j}||\right)}\right] \frac{\sum_{i} \frac{\pi_{i} v\left(\phi_{i}\right)}{\phi_{i}}}{\sum_{i} \pi_{i} v\left(\phi_{i}\right)},
$$

Lemma 1. FWLAN is stable iff $\rho(v)<1$.

$\diamond$

Capacity of system corresponding to scheduler $v$ is defined as that $\lambda=\lambda^{u}+\lambda^{d}$ beyond which the system is unstable. Capacity under $v$ is also called the stability region under $v$.

Theorem 1. Capacity is maximized by a scheduling policy $v_{c}^{*}\left(\phi_{1}\right)=1, v_{c}^{*}(\phi)=0$ for all $\phi \neq \phi_{1}$.

Proof : From (4), capacity is maximized if scheduler $\left\{v\left(\phi_{i}\right)\right\}$ minimizes $\frac{\sum_{i} \frac{\pi_{i} v\left(\phi_{i}\right)}{\phi_{i}}}{\sum_{i} \pi_{i} v\left(\phi_{i}\right)}$ or equivalently choose $u_{i}:=\frac{\pi_{i} v\left(\phi_{i}\right)}{\sum_{l} \pi_{l} v\left(\phi_{l}\right)}$ (with constraints $0 \leq u_{1} \leq 1$ for all $i$ and $\sum u_{i}=1$ ) to minimize $\sum_{i} u_{i} / \phi_{i}$, i.e, $u_{1}^{*}=1=1-u_{i}^{*}$ for all $i \neq 1$.

\subsection{Virtual Workload ${ }^{2}$}

The moments of the total service times of the customer, $\left\{\tilde{b}_{i}\right\},\left\{\tilde{b}_{i}^{(2)}\right\}$, who enters the system via queue $i$ can be cal-

\footnotetext{
${ }^{2}$ Virtual Workload : The total service time required by all the waiting customers $([4])$.
} 
culated using equations $2.2,2.3$ of [8] as:

$$
\begin{aligned}
\tilde{b}_{4 j-3} & =\tilde{b}_{4 j-2}=b_{4 j-3} \text { and } \tilde{b}_{4 j-1}=\tilde{b}_{4 j}=b_{4 j-1} \\
\tilde{b}_{\sigma} & =\eta E\left[\sum_{j=1}^{K} \frac{1_{\left\{L \in I_{j}\right\}}}{r\left(\left\|L-Q_{j}\right\|\right)}\right] \frac{\sum_{i} \frac{\pi_{i} v\left(\phi_{i}\right)}{\phi_{i}}}{\sum_{i} \pi_{i} v\left(\phi_{i}\right)}, \\
\tilde{b}_{4 j-3}^{(2)} & =\tilde{b}_{4 j-2}^{(2)}=b_{4 j-3}^{(2)} \text { and } \tilde{b}_{4 j-1}^{(2)}=\tilde{b}_{4 j}^{(2)}=b_{4 j-1}^{(2)} \\
\tilde{b}_{\sigma}^{(2)} & =\eta^{2} E\left[\sum_{j=1}^{K} \frac{1_{\left\{L \in I_{j}\right\}}}{r\left(\left\|L-Q_{j}\right\|\right)^{2}}\right] \frac{\sum_{i} \frac{\pi_{i} v\left(\phi_{i}\right)}{\phi_{i}^{2}}}{\sum_{i} \pi_{i} v\left(\phi_{i}\right)} .
\end{aligned}
$$

Using the Pseudo conservation laws (equations 6.4, 6.5) of [8] the virtual workload $\mathcal{V}_{\mathcal{W}}$ of the FWLAN is ( $W_{i}$ the waiting time at queue $i$ ) after some simplifications:

$$
\begin{aligned}
& \mathcal{V}_{\mathcal{W}}:=\sum_{i=1}^{\sigma} \tilde{b}_{i} \gamma_{i} E\left[W_{i}\right]=\sum_{i=1}^{\sigma} \frac{\lambda_{i} \tilde{b}_{i}^{(2)}}{2(1-\rho)}-\frac{\gamma_{i} b_{i}^{(2)}}{2}+ \\
& \rho \frac{c_{1} \sum_{j}\left\|Q_{j}-Q_{j-1}\right\|+K c_{2}}{2}+\frac{\sum_{i} \sum_{l} \gamma_{i} p_{i, l} \tilde{b}_{l} \sum_{k=i}^{l-1} w_{k}}{1-\rho} \\
& -\frac{\lambda^{u}\left(1-p_{s}\right)^{2}}{(1-\rho) p_{s}} \sum_{j} l_{j} \tilde{b}_{4 j} \sum_{k=4 j}^{4 j-1} w_{k}+ \\
& \frac{\sum_{j=1}^{K} w_{4 j}\left(\sum_{l=1}^{K} \lambda^{u} l_{l} \tilde{b}_{4 l} \sum_{k=4 l}^{4 j} \rho_{k} \lambda_{\sigma} \tilde{b}_{\sigma} \sum_{k=\sigma+1}^{4 j} \rho_{k}\right)}{1-\rho} .
\end{aligned}
$$

The expression for the virtual workload $\mathcal{V}_{\mathcal{W}}$ is complicated unlike that for the stability factor $\rho$. Thus it is difficult to obtain the analytical conclusion regarding the oppurtunistic policy that minimizes the virtual workload for a given system. However the expressions can be computed very easily and hence we study the optimal scheduling policy for a given system via some numerical examples. In these examples we obtain the optimal policy via exhaustive search.

We consider an example with three different fading states and symmetrical conditions, i.e., the position of arrival is uniform, stops are placed equidistant, uplink and downlink parameters are same etc. We set $\eta=1, \lambda^{u}=\lambda^{d}=0.0001$, $c_{1}\left\|Q_{1}-Q_{2}\right\|+c_{2}=1, E\left[1 / r(|Q-L|) \mid L \in I_{1}\right]=1$ and $E\left[1 / r(|Q-L|)^{2} \mid L \in I_{1}\right]=1.01$. In the table we consider various configurations of the fading states and their probabilities. The table shows optimal scheduler policy (the policy that minimizes the virtual workload $\mathcal{V}_{\mathcal{W}}$ ) for each configuration. We can see from the table that the policy which

\begin{tabular}{|c|c|c|}
\hline Fad factors & Probabilities & Optimal policy \\
\hline$\phi_{1}, \phi_{2}, \phi_{3}$ & $\pi_{1}, \pi_{2}, \pi_{3}$ & $v^{*}\left(\phi_{1}\right), v^{*}\left(\phi_{2}\right), v^{*}\left(\phi_{3}\right)$ \\
\hline$(1,0.4,0.01)$ & $(0.1,0.8,0.1)$ & $(1,1,0)$ \\
\hline$(1,0.4,0.01)$ & $(0.8,0.1,0.1)$ & $(1,0,0)$ \\
\hline$(0.5,0.4,0.3)$ & $(0.2,0.2,0.6)$ & $(1,1,1)$ \\
\hline$(1,0.9,0.03)$ & $(0.09,0.01,0.9)$ & $(1,1,0)$ \\
\hline
\end{tabular}

\section{Table 1: Optimal Scheduler for various systems}

maximizes the capacity (which we denote by MinCap) is not the best policy for minimizing the virtual workload (we denote the latter policy by MinW).

Let $\rho(u)$ denote the value of $\rho$ induced by a policy $u$. Any policy $u$ that serves with positive probability at some fading factor that is smaller than the best one, satisfies $\rho(u) / \rho($ MinCap $)>1$. Therefore, while performing better for given specific input rates for which it is designed, the stability region of MinW is strictly smaller than that of MinCap. For any arrival rates that are in the stability region of MinCap and not in those of MinW, the MinW schedule will give infinite expected virtual workload since it will fail to stabilize the system, where as MinCap will give finite expected virtual workload. We thus tradeoff here robustness with optimality at a particular value of input rate.

The above are only intial experiments and the main contribution of the paper is derivation of interesting performance measures for the FWLAN with oppurtunistic scheduler. In this paper we used the performance measures derived to obtain optimal scheduler. One can think of many more interesting uses of these performance measures like desiging optimal number of stops, designing optimal ferry path etc.

\section{Conclusions}

A wireless local area network facilitated with a moving message ferry, which oppurtunistically communicates with the nodes in the area is considered. The ferry based oppurtunistic LAN is mapped to a polling system (with rerouting) and important performance measures are derived. An optimal oppurtunistic scheduler which maximizes the capacity of the system or which minimizes the average virtual workload in FWLAN is discused. It is shown that the optimal scheduler which maximizes the capacity may not be optimal for average workload and vice versa.

\section{REFERENCES}

[1] E. Altman, P. Konstantopoulos, and Z. Liu, "Stability, Monotonicity and Invariant Quantities in General Polling Systems", Queuing Syst., 1992, vol. 11, no 1-2, pp. 35-57.

[2] Georgiadis, L. and Szpankowski, W., "Stability of Token Passing Rings", Queuing Syst., 1992, vol. 11, no. 1-2, pp. 7-33.

[3] O.J. Boxma, "Workloads and waiting times in single-server systems with multiple customer classes", Queuing Systems, 5 (1989) 185-214.

[4] O.J. Boxma, W.P. Groenendijk, "Pseudo-Conservation Laws in Cyclic-Service Systems", Journal of Applied Probability, Vol. 24, No. 4, Dec 1987, 949-964.

[5] O. J Boxma, H. Levy, U Yechiali, "Cyclic Reservation schemes for efficient operation of multiple-queue single-server systems", Annals of Operations Research, 1992, 187-208.

[6] S. Yousefi, E. Altman, R. El-Azouzi and M. Fathy, "Connectivity in vehicular ad hoc networks in presence of wireless mobile base-stations", in the Proceedings of the 7th International Conference on ITS Telecommunications, June 6-8, Sophia-Antipolis, France.

[7] Y. Shi and Y. T. Hou, "Theoretical results on base station movement problem for sensor network," in IEEE INFOCOM Š08, 2008.

[8] M. Sidi, H. Levy and S. W. Fuhrmann, "A queuing network with a single cyclically roving server", Queuing Systems 11, (special issue on Polling Models, Eds. H. Takagi and O. Boxma), pp.121-144, 1992.

[9] H. Takagi, "Analysis of Polling Systems", The MIT Press, 1986.

[10] M. M. B. Tariq, M. Ammar, and E. Zegura, "Message ferry route design for sparse ad hoc networks with mobile nodes", in Proc. of ACM MobiHoc, Florence, Italy, May 22-25, 2006, pp. 37-48. 